\title{
High-Power Single-Mode Simplified Antiresonant Reflecting Optical Waveguide (S-ARROW) Distributed Feedback Semiconductor Lasers
}

\author{
H. Yang, M. Nesnidal, A. Al-Muhanna, L. J. Mawst, Senior Member, IEEE, \\ D. Botez, Fellow, IEEE, T. A. Vang, F. D. Alvarez, and R. Johnson
}

\begin{abstract}
Simplified antiresonant-reflective-optical-waveguide distributed-feedback semiconductor lasers based on Al-free InGaAs-InGaAsP-InGaP materials are reported for the first time. Devices with $6.5-\mu \mathrm{m}$-wide emitting apertures operate single-frequency $(\lambda=0.968 \mu \mathrm{m})$ and single-spatial-mode to $157-\mathrm{mW}$ continuous-wave output power. The full-width at half-maximum of the lateral far-field pattern is $4.5^{\circ}$, in excellent agreement with theory. Relative intensity noise values as low as $-154 \mathrm{~dB} / \mathrm{Hz}$ are measured between $500 \mathrm{MHz}$ and $8 \mathrm{GHz}$.
\end{abstract}

Index Terms-Distributed feedback lasers, laser modes, semiconductor lasers.

\section{INTRODUCTION}

$\mathbf{H}$ IGH-POWER distributed-feedback (DFB) semiconductor lasers $(\lambda=0.98 \mu \mathrm{m})$, with stable operation over a wide temperature range, are attractive for pumping Er-doped optical fiber amplifiers as well as for high-fidelity RF-analog transmission. The radio-frequency (RF) optical link application has the additional requirement of operating with low relative intensity noise (RIN) so that the system noise is shotnoise limited. The Al-free InGaAs-InGaAsP-InGaP material system has been recently used to demonstrate record-high performance in $\mathrm{CW}$ output power and total power conversion efficiency: 10.6 -W continuous-wave (CW) [1], and $\eta_{p}=66 \%$ [2]; from broad-stripe (100- $\mu \mathrm{m}$ wide) diode lasers. For the wavelength region of interest, $\lambda \sim 0.9-1.0 \mu \mathrm{m}$, the advantages of the Al-free material system over the conventional InGaAs-AlGaAs-based materials have been well established [1]-[3]: 1) lower device series resistance and 2) the low surface oxidation of InGaP compared with $\mathrm{AlGaAs}$, greatly simplifies regrowth. Thus, novel index-guided laser structures which require multiple growth steps, such as, phase-locked antiguided arrays [4], triple-core antiresonant-reflective-optical waveguide (TCA) structures [3], [5], and distributed feedback (DFB) [6] lasers have been reported that exploit the advantages of Al-free materials.

In this letter, we report the first demonstration of a novel diode laser structure: the simplified antiresonant reflecting

Manuscript received March 3, 1998; revised April 23, 1998.

H. Yang, A. Al-Muhanna, L. J. Mawst, and D. Botez are with Reed Center for Photonics, University of Wisconsin at Madison, Madison, WI 53706 USA.

M. Nesnidal was with Reed Center for Photonics, University of Wisconsin at Madison, Madison, WI 53706 USA. He is now with TRW Inc., Redondo Beach, CA 90278 USA.

T. A. Vang, F. D. Alvarez, and R. Johnson are with TRW Inc., Redondo Beach, CA 90278 USA.

Publisher Item Identifier S 1041-1135(98)05363-4. optical waveguide (S-ARROW) laser [7]; which also incorporates a distributed-feedback (DFB) grating. Conventional ARROW-type diode lasers have previously demonstrated stable operation in a single-spatial mode to high-output powers (0.5-W pulsed [8], 0.3-W CW [9]) from 4 to $6-\mu \mathrm{m}$-wide aperture devices. The ARROW-type structures exhibit a large built-in lateral (negative) index step $(\Delta n \sim 0.05)$, which provides mode stability to high-output power, as well as strong discrimination against higher order lateral modes. In the SARROW structure, the outer low-index reflecting region is eliminated, proving a simpler structure with strong stability against gain-spatial-hole burning. [7]

The complete laser structure is shown schematically in Fig. 1. In the longitudinal direction, a second-order DFB grating buried beneath the quantum-well active region provides single-frequency operation. The use of a second-order grating provides first-order (surface) radiation losses which enhance mode selectivity [10], and leads to a higher yield of single-frequency devices. As described below, the use of a lower grating (i.e., below the active layer) allows the lateral antiguide (S-ARROW) structure to be fabricated by etch and regrowth above the active layer. The lateral SARROW waveguide consists of a central low-index core region (6.5- $\mu \mathrm{m}$ wide) surrounded by high-index quarter-wave $\left(\lambda_{1} / 4\right.$, where $\lambda_{1}=$ lateral (projected) wavelength in highindex regions) antiresonant reflecting regions [7]. As a result, the fundamental S-ARROW (leaky) mode experiences lowedge (lateral) radiation losses. At the same time, higher order modes, which are not antiresonant, suffer excessive radiation losses, preventing them from reaching laser threshold. An above threshold analysis, previously reported [7], demonstrates that the S-ARROW structure is virtually immune to gainspatial-hole burning (GSHB), allowing a single-lateral mode to be maintained to drive currents in excess of $10 \times$ threshold. In addition, the S-ARROW structure is advantageous from a fabrication standpoint, because of the relatively large parameter window (in terms of lateral index step as described below) over which single-mode operation can be obtained.

\section{Device Structure and Fabrication}

The S-ARROW-DFB laser structure was grown by a threestep low-pressure metal-organic chemical vapor deposition (LP-MOCVD) process on a (100) $0.5^{\circ}$-off toward (110) $\mathrm{n}^{+}$GaAs substrate. The first growth consists of a $0.5-\mu \mathrm{m}$-thick 


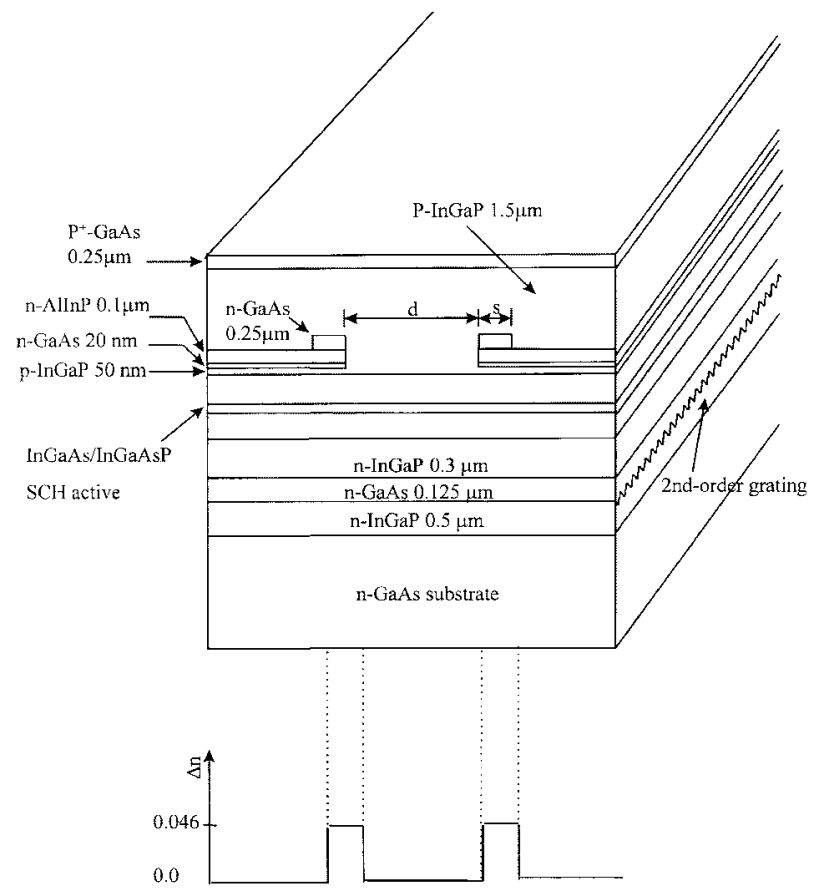

Fig. 1. Schematic view of the layered structure for the S-ARROW DFB laser and lateral effective index variation.

n-InGaP lower cladding layer, into which a second-order DFB grating with a grating period of $2880 \AA$ and tooth height of $750 \AA$ is formed by using conventional holography and wet chemical etching ( $\mathrm{HBr}$ system). In the second growth step, the InGaP grating is planarized (confirmed by TEM crossections) with a $0.125-\mu \mathrm{m}$-thick $\mathrm{n}$-GaAs layer, followed by a $0.3-\mu \mathrm{m}$-thick $n$-InGaP spacer layer, and a $0.4-\mu \mathrm{m}$-thick $\operatorname{InGaAsP}\left(E_{g}=1.62 \mathrm{eV}\right)$ separate-confinement heterostructure $(\mathrm{SCH})$ with a double-quantum-well (DQW) InGaAs-active region, similar to those previously reported [1], [2]. Additional layers consisting of a GaAs etch-stop layer, an AlInP currentblocking layer, and the high-index $(0.25-\mu \mathrm{m}$-thick) GaAs guide layer, as shown in Fig. 1, are grown above the $\mathrm{SCH}$ region during the second growth step. The S-ARROW pattern is then defined by conventional photolithography and wet chemically etched, prior to the final growth step. The third growth consists of a $1.5 \mu \mathrm{m}$-thick p-InGaP upper cladding layer and $0.25-\mu \mathrm{m}$-thick $\mathrm{p}^{+}-\mathrm{GaAs}$ contact layer. The resulting lateral structure consists of a $6.5-\mu \mathrm{m}$-wide low-index core region, clad by high-index optical reflecting layers of width $s(s \sim 0.85 \mu \mathrm{m})$ and low-index terminations. The lateral effective-index step is designed to be $\Delta n=0.046$, so that the reflector regions are antiresonant for the fundamental SARROW mode. The injected current is self-aligned to the low-index core region by the n-AlInP-GaAs reverse-biased junctions (i.e., current blocking layers). The thicknesses of the blocking layers are chosen such that the effective refractive index in the outer low-index terminations is identical to that in the low-index core region. Thus one obtains the lateral effective-index profile shown in Fig. 1. After contact metallizations are completed, 500- $\mu \mathrm{m}$-long devices are facet coated (5\%/95\%) and mounted junction-side down on copper heatsinks for $\mathrm{CW}$ testing.

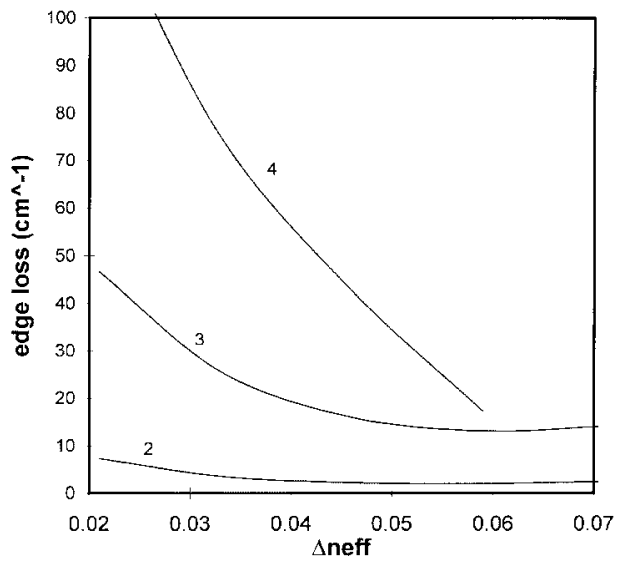

Fig. 2. Calculated lateral radiation losses for the S-ARROW modes $2-4$ as a function of lateral index-step $\left(\Delta n_{\text {eff }}\right)$ corresponding to the structure shown in Fig. 1.

The ARROW modes of the complete two-dimensional (2-D) waveguide, formed by the layered structure shown in Fig. 1, were analyzed using a rigorous transverse mode-matching technique [11]. The dominant mechanism for high-order mode discrimination in the ARROW structure is lateral edge radiation losses. Fig. 2 shows the calculated modedependent edge radiation losses versus the effective index step, $\Delta n_{\text {eff }}$, for structures with $d=6 \mu \mathrm{m}$ (low-index core) and $s=0.9 \mu \mathrm{m}$ (high-index reflectors). The antiresonant point $\left(\Delta n_{\mathrm{eff}}=0.046\right)$, corresponds to the minimum edge loss for the fundamental S-ARROW mode (mode number 2). The mode number corresponds to the number of intensity nulls in the optical intensity profile [11]. It should be noted that value of $\Delta n_{\mathrm{eff}}$ at antiresonance, determined by the 2-D calculation, differs from that calculated [8] using an effectiveindex approximation $\left(\Delta n_{\mathrm{eff}}=0.01\right)$, assuming the reflector width is equal to one-quarter lateral wavelength (i.e., $\lambda_{1} / 4$ ). The large difference in edge losses $\left(2.2 \mathrm{~cm}^{-1}\right.$ for mode 2 versus $16 \mathrm{~cm}^{-1}$ for mode 3 ) between the fundamental mode and the high-order modes and negligible GSHB [7] can effectively suppress the high-order modes, allowing the device to operate to high-output power in a single spatial mode. Fig. 2 also shows that large mode discrimination exists over a wide effective-index step range, which demonstrates the loose fabrication tolerances associated with the S-ARROW structure.

\section{DeVICE Results}

The measured CW single-frequency $(\lambda \sim 0.968 \mu \mathrm{m})$ spectral characteristics at room temperature are shown in Fig. 3, for injection currents of $300 \mathrm{~mA}(61 \mathrm{~mW}), 400$ $\mathrm{mA}(127 \mathrm{~mW})$, and $450 \mathrm{~mA}(157 \mathrm{~mW})$, respectively. DFB operation is confirmed from the measured spectral shift with temperature $\left(0.06 \mathrm{~nm} /{ }^{\circ} \mathrm{C}\right)$, with a coupling coefficient and cavity length product $\kappa L$ of 1.5 , estimated [12] from the measured subthreshold spectra. At higher output powers, the onset of multiple-longitudinal modes broaden the spectra. Threshold currents are in the 100-125-mA range. Lower threshold currents can be expected by optimizing the coupling coefficient, since Fabry-Perot S-ARROW structures of similar 


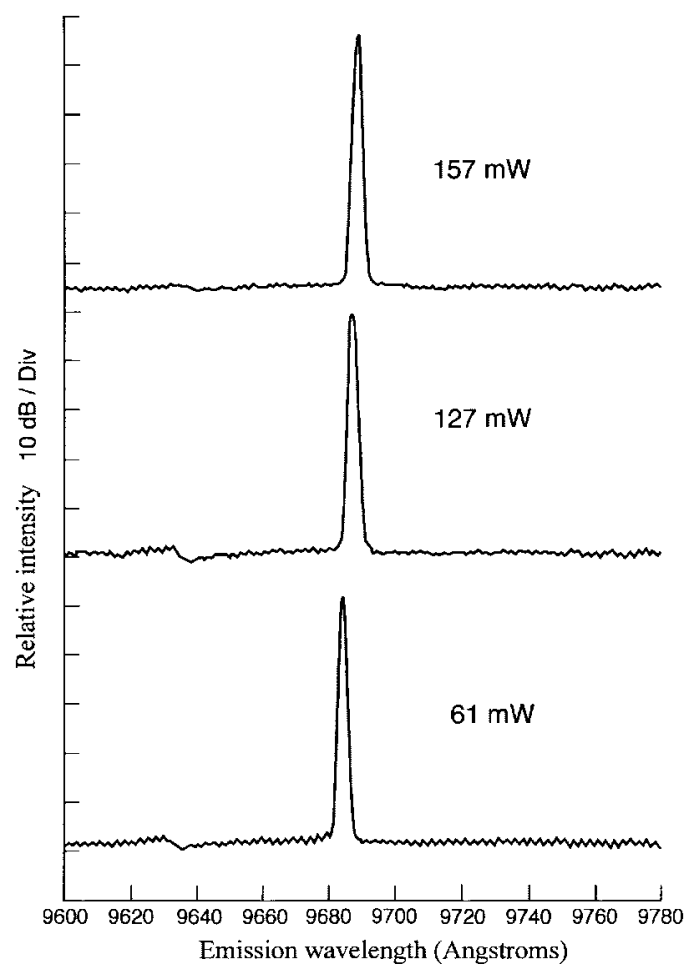

Fig. 3. Measured spectral characteristics for the S-ARROW DFB laser as a function of $\mathrm{CW}$ output power.

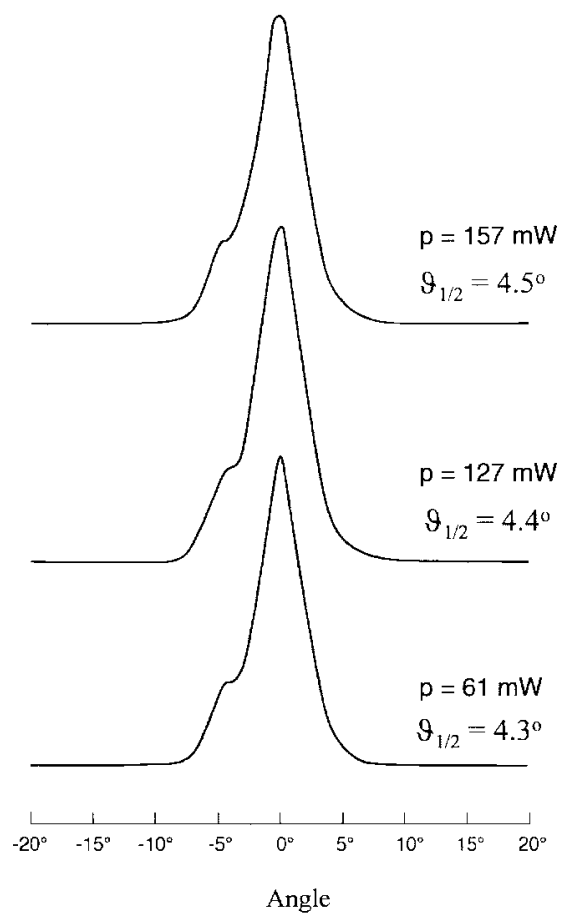

Fig. 4. Measured lateral far-field radiation pattern as a function of $\mathrm{CW}$ output power for the S-ARROW DFB laser.

geometry have exhibited threshold currents as low as $20 \mathrm{~mA}$. The results in Fig. 3 represent record-high single-mode $\mathrm{CW}$ output powers for DFB lasers in the 980-nm wavelength region. Previously reported [6] Al-free index-guided DFB lasers have operated to $58-\mathrm{mW} \mathrm{CW}$ output power.
The measured lateral far-field beam pattern as a function of CW output power is shown in Fig. 4. Stable, fundamental S-ARROW mode operation is observed up to the maximum single-frequency CW output power, $157 \mathrm{~mW}$. The FWHM of the beam pattern at $157 \mathrm{~mW}$ is $4.5^{\circ}$, in excellent agreement with theory $\left(4.5^{\circ}\right.$ for $6.5-\mu \mathrm{m}$-wide core $)$. Calculations indicate that the asymmetrical shoulder observed on the measured far-field, shown in Fig. 4, is due to a deviation of the photolithography alignment $( \pm 0.2 \mu \mathrm{m})$, which results in unequal widths of the two quarter-wave reflectors. High-output-power, single-mode operation is expected to result in low RIN, which is desirable for RF-Photonic applications. RIN measurements on the S-ARROW DFB lasers from $500 \mathrm{MHz}$ to $8 \mathrm{GHz}$ indicate values as low as $-154 \mathrm{~dB} / \mathrm{Hz}$.

\section{CONCLUSION}

Al-free InGaAs-InGaAsP-InGaP-based S-ARROW-DFB diode lasers have been demonstrated by three-step MOCVD growth. Devices operate CW with single-frequency and singlespatial mode up to $157 \mathrm{~mW}$ output power. Measurements of RIN between $500 \mathrm{MHz}$ and $8 \mathrm{GHz}$ yield values as low as -154 $\mathrm{dB} / \mathrm{Hz}$. Higher output powers can be expected by optimization of the $\kappa L$, facet-reflectivities, and spectral gain-peak position relative to the Bragg frequency.

\section{REFERENCES}

[1] A. Al-Muhanna, L. J. Mawst, D. Botez, D. Garbuzov, R. V. Martinelli, and J. Connolly, "10.6 W CW front-facet power from 100 $\mu \mathrm{m}$-aperture $0.97 \mu \mathrm{m}$-emitting Al-free diode lasers," in Tech. Dig. IEEE/OSA CLEO'98, San Francisco, CA, May 3-7, 1998, vol. 6, pp. $11-12$.

[2] D. Botez, L. J. Mawst, A. Bhattacharya, J. Lopez, J. Li, T. F. Kuech, V. P. Iakovlev, G. I. Suruceanu, A. Caliman, and A. V. Syrbu, " $66 \%$ CW wallplug efficiency from Al-free $0.98 \mu$ m-emitting diode lasers," Electron. Lett., vol. 32, pp. 2012-2013, 1996.

[3] C. Zmudzinski, D. Botez, L. J. Mawst, A. Bhattacharya, and M Nesnidal, "Three-core ARROW-type diode laser: Novel high-power, single-mode device, and effective master oscillator for flared antiguided MOPA's," IEEE J. Select. Topics Quantum Electron., vol. 1, pp. 129-135, June 1995.

[4] H. Yang, L. J. Mawst, M. Nesnidal, J. Lopez, A. Bhattacharya, and D. Botez, " $10 \mathrm{~W}$ near-diffraction-limited peak pulsed power from A-free, $0.98 \mu \mathrm{m}$-emitting phase-locked antiguided array," Electron. Lett., vol. 33, pp. 136-137, 1997.

[5] L. J. Mawst, A. Bhattacharya, M. Nesnidal, J. Lopez, D. Botez, A. V. Syrbu, V. P. Ykovlev, G. I. Suruceanu, A. Z. Mereutza, M. Jansen, and R. F. Nabiev, " MOVPE-grown high CW power InGaAs/InGaAsP/InGaP diode lasers," J. Cryst. Growth, vol. 170, pp. 383-389, 1997.

[6] Y. K. Sin and H. Horikawa, "High power $0.98 \mu \mathrm{m} \mathrm{InGaAs-GaAs-}$ InGaP distributed feedback buried heterostructure strained quantum well lasers," Electron. Lett., vol. 29, pp. 920-922, 1993.

[7] I. V. Goltser, L. J. Mawst, and D. Botez, "Single-cladding antiresonant reflecting optical waveguide-type diode lasers," Opt. Lett., vol. 20, pp. 2219-2221, 1995.

[8] L. J. Mawst, D. Botez, C. Zmudzinski, and C. Tu, "Design optimization of ARROW-type diode lasers," IEEE Photon. Technol. Lett., vol. 4, pp. 1204-1206, 1992.

[9] _, $0.3 \mathrm{~W} \mathrm{CW}$ single-spatial-mode operation from large-core ARROW-type diode lasers," Electron. Lett., vol. 28, pp. 1793-1795, 1992.

[10] R. F. Kazarinov and C. H. Henry, "Second-order distributed feedback lasers with mode selection provided by first-order radiation losses," IEEE J. Quantum Electron., vol. 21, pp. 144-150, 1985.

[11] D. Botez, "High-power monolithic phase-locked arrays of antiguided semiconductor diode lasers," IEE Proc. J., vol. 139, pp. 14-23, 1992.

[12] K. Iga, "On the use of effective refractive index in DFB laser mode separation," Jpn. J. Appl. Phys., vol. 22, p. 1630, 1983. 